\title{
ANÁLISIS DE CORRELACIÓN CANÓNICA ENTRE VOICE ONSET TIME Y DIADOCOCINESIA DEL HABLA DEL ESPAÑOL COLOMBIANO
}

\section{Canonical Correlation Analisys foroice Onset Time and Speech Diadoco- kinesia in colombian spanish}

Heriberto Jose Rangel Navia ${ }^{(1)}$, Juan José Pabón Villamizar, Diana Carolina Martinez Siza, Karent Susana Contreras Suarez.

\section{RESUMEN}

INTRODUCCIÓN: Evaluar a través del análisis de correlación canónica (ACC) la existencia de relaciones predictivas para uso diagnóstico entre Voice Onset Time (VOT) y Diadococinesia del Habla (DDK) en el español colombiano. MÉTODOS: Estudio transversal con inclusión población aleatoria por muestreo estratificado. 69 participantes 22 hombres, 44 mujeres. Recolección de datos por repetición de 19 palabras para el cálculo de VOT y repetición silábica para DDK. EI ACC fue usado como estrategia para la evaluación estadística de correlaciones entre los datos. RESULTADOS: La media de VOT por sonido fue de /p/ 16,74 seg; /t/ 18,57 seg; /k/ 25,59 seg; /b/ -33,66 seg; /d/ -21,28 seg; /g/ -24,84 seg. Para DDK los valores obtenidos fueron /pa/ 17.08 repeticiones por segundo (rps); /ta/ 17.12rps; /ka/ $14.96 \mathrm{rps}$; /pataka/ $15.92 \mathrm{rps}$. No se encontro correlación canónica entre VOT y DDK. ANÁLISIS Y DISCUSIÓN: La ausencia de relaciones predictivas entre VOT y DDK podría explicarse por efecto de la tasa de producción del habla como elemento central en la producción de la DDK, o por efecto de la composicion de la relación de sentido entre DDK y VOT necesaria para el ACC. CONCLUSIONES: No existe relación predictiva entre VOY y DDK, sin embargo exuste entre estos una relación de singularidades no positivas a la cual no es sensible el ACC.

PALABRAS CALVE: Habla, Acústica, Diagnostico

\section{ABSTRACT}

INTRODUCTION: Evaluate through canonical correlation analysis (CCA) the existence of predictive relationships for diagnostic use between Voice Onset Time (VOT) and Speech Diadochokinesia (DDK) in Colombian Spanish. METHODS: Cross-sectional study with random including and stratified sample population. 69 participants men 22, 44 women. Data collection by repeating 19 words for calculating VOT and syllabic repetition to DDK. The CCA was used as a strategy for statistical evaluation of correlations between data. RESULTS: Mean Sound was VOT /p/ $16,74 \mathrm{sec} ; / \mathrm{t} / 18,57 \mathrm{sec}$ / /k/ 25,59 sec; /b/ -33,66 sec; /d/ -21,28 sec; /g/ -24,84 sec. For DDK the values obtained were /pa/ 17.08 repetitions per second (rps); /ta/ $17.11 \mathrm{rps}$; /ka/ $14.96 \mathrm{rps}$; /pataka/ 15,92 rps. No canonical correlation between VOT and DDK was found ANALYSIS AND DISCUSSION: he lack of predictive relationships between VOT and DDK could be explained by the effect of the rate of speech production as a central element in the production of the DDK, or effect of the composition of the relationship of meaning between DDK and VOT necessary for the CCA CONCLUSIONS: There is no predictive relationship between VOY and DDK, however exuste a relationship between these non-positive singularities which is not sensitive CCA

KEYWORDS: Speech, Acoustics, Diagnosis,

(1) Fonoaudiólogo, Magister en educación, Candidato a Doctor en Educación. Docente Universidad de Pamplona . 


\section{INTRODUCCIÓN}

La diadococinesia del habla (DDK) y el Voice Onset Time (VOT), son usados como índices acústicos, articulatorios y de coordinación neuromotora para el diagnóstico y monitoreo de los desórdenes motores del habla (1), (2), (3), (4), (5), (6), (7), (8), (9), (10), particularmente en el estudio de las disartrias, (2), (3), (4), (5) , (9), (7), sin embargo, el estudio de las relaciones internas de estos índices a nivel matemático o estadístico, no son igualmente frecuentes, al menos desde la clínica, pues en lo tocante al desarrollo de estrategias de machine learning o de sistemas de automatización y control este tipo de búsquedas constituyen un tema emergente (9), (10), (11). Este trabajo busca desde la primera perspectiva (Clínica) evaluar a través del análisis de correlación canónica la existencia de relaciones predictivas para el uso diagnóstico entre VOT y DDK en el español colombiano.

El Análisis de Correlación Canónica (ACC) permite identificar relaciones lineares entre dos conjuntos de variables diferentes pero al mismo tiempo relacionados entre sí a través de al menos una unidad de sentido (12), este tipo de composición permite el uso de la ACC como estrategia para la evaluación de relaciones predictivas entre VOT y DDK, a saber; (a) EI VOT es una medida de tiempo relacionada con las transformaciones del tracto vocal durante la producción de sonidos oclusivos sordos o sonoros (1), (5), (8); (b) La DDK es una medida de coordinación neuromuscular basada en el tiempo por efecto de la repetición secuencial o alternativa de sonidos oclusivos sordos (6), (3), (2), (9); (c) El VOT y la DDK comparten una composición acústica básica; sonidos oclusivos sordos; pero las estrategias de análisis clínico para cada uno de ellos son diferentes.

Así las cosas, el VOT y las DDK comparten como unidad de sentido la composición acústica y articulatoria de los sonidos oclusivos sordos, mientras que se diferencian entre sí a partir del objeto clínico del análisis, para el primero es el tiempo como índice acústico de transición articulatoria, en el segundo el foco de interpretación es el número de repeticiones por segundo como índice de control neuromuscular del tracto vocal. En este sentido es posible hipotetizar que el VOT puede ser un predictor del máximo número de repeticiones por segundo de la DDK, toda vez que a mayor duración del VOT las transiciones y duración total de los sonidos oclusivos emitidos podría ser menor, evento que afectaría negativamente a la DDK.

No obstante, el propósito final de la DDK no es la producción inteligible del sonido, sino el control de la enervación muscular del tracto vocal, visible acústicamente a través de la barra de explosión de las oclusivas sordas (1), (8), evento que podría poner énfasis no en la composición articulatoria del sonido, sino en la velocidad de su producción, por lo que sería igualmente posible hipotetizar que entre VOT y DDK no existiría relación alguna más allá del uso común del custer consonántico oclusivo sordo.

\section{MÉTODOS}

Este trabajo se diseñó como un estudio descriptivo transversal. El universo poblacional estudiado correspondió a 2092 personas, el muestreo utilizado para desarrollar la recolección de datos fue de tipo estratificado para garantizar la participación equitativa de todos los segmentos poblacionales, alcanzando un total de 68 participantes en el estudio 44 mujeres, 22 hombres. Los criterios de inclusión y exclusión usados fueron;

1. Criterios de Inclusión

a. Pertenecer al universo poblacional

b. Ser mayor de edad

c. Otorgar consentimiento informado a los investigadores para el uso de los datos en el estudio

2. Criterios de Exclusión 
a. Haber cursado o estar cursando tratamiento ortodoncia

b. Haber sufrido trauma de cabeza, cuello o clavícula

c. Haber sufrido algún tipo de parálisis facial

d. Presentar mal oclusión frontal o lateral al momento del estudio

Todos los datos acústicos se recolectaron en una cámara sonoamortiguada con un micrófono Shure SM48 y un preamplificador Shure X2s, se utilizó el software Speech Analizer 3.1 para la grabación y análisis de DDK, adicionalmente de usó el programa de computo Pratt para el procesamiento del VOT.

La recolección de datos para DDK siguió las recomendaciones de Rangel, Guerrero y Navarro (6), para ello las repeticiones silábicas fueron catalogadas en: (a) Tasa de Movimientos Secuanciales del Habla (TMSH) para las silabas /pa/, /ta/ y / ka/; y (b) Tasa de Movimientos Alternativos del Habla (TMAH) para el enunciado /pataka/. Los datos de VOT correspondieron a 19 palabras que contenían sonidos oclusivos sordos y sonoros, ver Tabla 00. El ACC se realizó usando el paquete R Project for Statistical Computing.

\section{RESULTADOS}

Los resultados del estudio se encuentran categorizados en dos dimensiones; a saber, (a) Análisis de Tendencia Central para VOT y DDK; y (b) ACC VOT-DDK. La primera capa permitirá comparar los resultados del estudio con otros disponibles, y la segunda dar cuenta del propósito central de este trabajo.

(a) Análisis de Tendencia Central para VOT y DDK

\begin{tabular}{llllllll}
\hline V.O.T SONORAS & & & & & \\
V.O.T & B & & & & D & G \\
\hline MEDIA & BUFAN- & BOLSO & BARCO & TAMBOR & BUFAN- & DIENTE & GORRO \\
& DA & & & & DA & & \\
\cline { 2 - 7 } & -22.069 & -44.093 & -33.631 & -46.87 & -23.92 & -19.24 & -24.84 \\
\hline MEDIA- & -29.008 & -49.790 & -41.277 & -47.102 & -32.615 & -18.308 & -23.430 \\
NA & & & & & & & \\
\hline
\end{tabular}

Fuente: Los autores

TABLA 1: resultados de media y mediana de V.O.T sonoras

\begin{tabular}{|c|c|c|c|c|c|c|c|c|}
\hline \multicolumn{9}{|c|}{ MEDIANA V.O.T SONORAS } \\
\hline \multirow[t]{2}{*}{ EDAD } & \multirow{2}{*}{$\begin{array}{l}\text { GENE- } \\
\text { RO }\end{array}$} & \multicolumn{4}{|l|}{$\mathrm{B}$} & \multicolumn{2}{|l|}{$\mathrm{D}$} & \multirow{2}{*}{$\frac{G}{\text { GORRO }}$} \\
\hline & & $\begin{array}{l}\text { BUFAN- } \\
\text { DA }\end{array}$ & BOLSO & BARCO & $\begin{array}{l}\text { TAM- } \\
\text { BOR }\end{array}$ & $\begin{array}{l}\text { BUFAN- } \\
\text { DA }\end{array}$ & DIENTE & \\
\hline \multirow[t]{2}{*}{17} & $\mathrm{~F}$ & -49.56 & -36.82 & -18.91 & -45.46 & -35.73 & -17.29 & -13.00 \\
\hline & $M$ & No data & No data & No data & No data & No data & No data & No data \\
\hline \multirow[t]{2}{*}{18} & $\mathrm{~F}$ & -31.65 & -57.95 & -29.92 & -47.66 & -47.99 & -18.27 & -21.100 \\
\hline & 18 & -56.48 & -47.00 & -27.69 & -50.21 & -15.11 & -20.22 & -24.00 \\
\hline \multirow[t]{2}{*}{19} & $\mathrm{~F}$ & -36.41 & -59.98 & -31.91 & -45.24 & -19.75 & -9.56 & -24.77 \\
\hline & $M$ & -49.73 & -54.67 & -33.45 & -48.81 & -35.07 & -25.26 & -24.84 \\
\hline \multirow[t]{2}{*}{20} & $\mathrm{~F}$ & -9.50 & -43.51 & -24.31 & -48.15 & -15.47 & -17.39 & -17.65 \\
\hline & $M$ & -25.88 & -68.25 & -29.13 & -48.24 & -28.85 & -15.13 & -17.97 \\
\hline
\end{tabular}


Rangel Navia H , Pabón Villamizar J, Martinez Siza D, Contreras Suarez K.

\begin{tabular}{lllllllll}
\hline 21 & $F$ & -18.09 & -38.94 & -48.80 & -46.11 & -20.23 & -22.53 & -27.34 \\
\cline { 2 - 8 } & $M$ & -16.70 & -40.38 & -34.88 & -46.31 & -23.92 & -23.78 & -17.87 \\
\hline 22 & $F$ & No data & No data & No data & No data & No data & No data & No data \\
\cline { 2 - 8 } & $M$ & No data & No data & No data & No data & No data & No data & No data \\
\hline 23 & $F$ & -9.37 & -37.84 & -23.34 & -50.34 & -11.73 & -11.17 & -18.85 \\
\cline { 2 - 8 } & $M$ & -47.543 & -36.978 & -29.582 & -45.27 & -52.83 & -20.639 & -29.582 \\
\hline 24 & $F$ & -37.24 & -49.04 & -22.25 & -51.74 & -50.51 & -16.22 & -29.73 \\
\hline 25 & $M$ & -34.71 & -46.288 & -17.886 & -53.88 & -20.25 & -17.357 & -17.43 \\
\hline 26 & $F$ & No data & No data & No data & No data & No data & No data & No data \\
\cline { 2 - 8 } & $\mathrm{M}$ & -40.78 & -44.093 & -16.557 & -42.64 & -60.47 & -19.383 & -26.598 \\
\hline & $\mathrm{F}$ & -30.28 & -79.03 & -56.04 & -43.29 & -32.84 & -15.34 & -12.59 \\
\hline
\end{tabular}

Fuente: Los autores

TABLA 2: resultados de la comparativos V.O.T mediana según género y edad.

\begin{tabular}{|c|c|c|c|c|c|c|c|c|}
\hline \multicolumn{9}{|c|}{ MEDIA V.O.T SONORAS } \\
\hline \multirow[t]{2}{*}{ EDAD } & \multirow{2}{*}{$\begin{array}{l}\text { GENE- } \\
\text { RO }\end{array}$} & \multicolumn{4}{|l|}{$\mathrm{B}$} & \multicolumn{2}{|l|}{$\mathrm{D}$} & \multirow{2}{*}{$\frac{G}{\text { GORRO }}$} \\
\hline & & $\begin{array}{l}\text { BUFAN- } \\
\text { DA }\end{array}$ & BOLSO & BARCO & $\begin{array}{l}\text { TAM- } \\
\text { BOR }\end{array}$ & $\begin{array}{l}\text { BUFAN- } \\
\text { DA }\end{array}$ & DIENTE & \\
\hline \multirow[t]{2}{*}{17} & $\mathrm{~F}$ & -49.56 & -36.82 & -18.91 & -45.46 & -35.73 & -17.29 & -13.00 \\
\hline & $M$ & No data & No data & No data & No data & No data & No data & No data \\
\hline \multirow[t]{2}{*}{18} & $\mathrm{~F}$ & -30.13 & -61.49 & -30.10 & -47.93 & -35.88 & -30.10 & -21.51 \\
\hline & $M$ & -56.48 & -47.00 & -27.69 & -50.21 & -15.11 & -20.22 & -24.00 \\
\hline \multirow[t]{2}{*}{19} & $\mathrm{~F}$ & -40.17 & -51.43 & -40.69 & -45.57 & -28.84 & -12.86 & -23.42 \\
\hline & $M$ & -37.68 & -57.24 & -30.35 & -48.85 & -36.0 & -18.72 & -23.83 \\
\hline \multirow[t]{2}{*}{20} & $\mathrm{~F}$ & -13.80 & -48.47 & -30.29 & -48.01 & -22.41 & -17.87 & -17.29 \\
\hline & $M$ & -32.24 & -59.62 & -42.75 & -48.34 & -26.57 & -15.95 & -18.81 \\
\hline \multirow[t]{2}{*}{21} & $\mathrm{~F}$ & -24.50 & -45.27 & -50.07 & -45.91 & -33.07 & -20.48 & -26.24 \\
\hline & $M$ & -27.93 & -51.35 & -45.87 & -48.11 & -33.66 & -21.35 & -21.75 \\
\hline \multirow[t]{2}{*}{22} & $\mathrm{~F}$ & No data & No data & No data & No data & No data & No data & No data \\
\hline & $M$ & No data & No data & No data & No data & No data & No data & No data \\
\hline \multirow[t]{2}{*}{23} & $\mathrm{~F}$ & -9.37 & -37.84 & -23.34 & -50.34 & -11.73 & -11.17 & -18.85 \\
\hline & $M$ & -47.543 & -36.978 & -29.582 & -45.27 & -52.83 & -20.639 & -29.582 \\
\hline \multirow[t]{2}{*}{24} & $\mathrm{~F}$ & -37.24 & -49.04 & -22.25 & -51.74 & -50.51 & -16.22 & -29.73 \\
\hline & $M$ & -34.71 & -46.288 & -17.886 & -53.88 & -20.25 & -17.357 & -17.43 \\
\hline \multirow[t]{2}{*}{25} & $\mathrm{~F}$ & No data & No data & No data & No data & No data & No data & No data \\
\hline & $M$ & -40.78 & -44.093 & -16.557 & -42.64 & -60.47 & -19.383 & -26.598 \\
\hline \multirow[t]{2}{*}{26} & $\mathrm{~F}$ & -30.28 & -79.03 & -56.04 & -43.29 & -32.84 & -15.34 & -12.59 \\
\hline & $M$ & No data & No data & No data & No data & No data & No data & No data \\
\hline
\end{tabular}

Fuente: Los autores

TABLA 3: resultados de la comparativos V.O.T media según género y edad. 


\begin{tabular}{|c|c|c|c|c|c|c|c|}
\hline \multicolumn{8}{|c|}{ GENERAL V.O.T SORDOS } \\
\hline \multicolumn{8}{|c|}{$P$} \\
\hline & LAPIZ & PEINE & & PIEDRA & & ESPADA & \\
\hline $\begin{array}{l}\text { MEDIA- } \\
\text { NA }\end{array}$ & 17.23 & 14.71 & & 18.73 & & 15.846 & \\
\hline MEDIA & 17.361 & 14.653 & & 18.490 & & 16.494 & \\
\hline \multicolumn{8}{|c|}{$\mathrm{T}$} \\
\hline & TAZA & TAMBOR & FRUTA & DIENTE & $\begin{array}{l}\text { CHA- } \\
\text { QUETA }\end{array}$ & $\begin{array}{l}\text { AUTO- } \\
\text { BUS }\end{array}$ & CRISTAL \\
\hline $\begin{array}{l}\text { MEDIA- } \\
\text { NA }\end{array}$ & 14.765 & 15.578 & 12.89 & 18.093 & 32.37 & 16.229 & 16.558 \\
\hline MEDIA & 16.544 & 16.010 & 13.596 & 18.385 & 32.849 & 15.555 & 17.102 \\
\hline \multicolumn{8}{|c|}{$\mathrm{K}$} \\
\hline & CARA & BARCO & MOSCA & BLANCO & \multicolumn{3}{|c|}{ CHAQUETA } \\
\hline $\begin{array}{l}\text { MEDIA- } \\
\text { NA }\end{array}$ & 24.80 & 28.454 & 21.667 & 31.658 & \multicolumn{3}{|c|}{14.628} \\
\hline MEDIA & 23.407 & 32.248 & 22.260 & 33.953 & \multicolumn{3}{|c|}{16.125} \\
\hline
\end{tabular}

Fuente: Los autores

TABLA 4: resultados de media y mediana de V.O.T sordas.

\begin{tabular}{|c|c|c|c|c|c|c|c|}
\hline \multicolumn{8}{|c|}{ V.O.T SORDAS } \\
\hline \multirow[t]{2}{*}{ EDAD } & \multirow[t]{2}{*}{ GENERO } & & \multicolumn{5}{|l|}{$\mathrm{K}$} \\
\hline & & & CARA & BARCO & MOSCA & BLANCO & $\begin{array}{l}\text { CHA- } \\
\text { QUETA }\end{array}$ \\
\hline \multirow[t]{4}{*}{17} & \multirow[t]{2}{*}{$\mathrm{F}$} & MEDIA & 27.34 & 41.82 & 26.41 & 30.79 & 34.28 \\
\hline & & $\begin{array}{l}\text { MEDIA- } \\
\text { NA }\end{array}$ & 27.34 & 41.82 & 26.41 & 30.79 & 34.28 \\
\hline & \multirow[t]{2}{*}{$M$} & MEDIA & No data & No data & No data & No data & No data \\
\hline & & $\begin{array}{l}\text { MEDIA- } \\
\text { NA }\end{array}$ & No data & No data & No data & No data & No data \\
\hline \multirow[t]{4}{*}{18} & \multirow[t]{2}{*}{$\mathrm{F}$} & MEDIA & 24.80 & 29.82 & 17.14 & 32.37 & 33.01 \\
\hline & & $\begin{array}{l}\text { MEDIA- } \\
\text { NA }\end{array}$ & 24.50 & 33.44 & 18.57 & 34.42 & 33.99 \\
\hline & \multirow[t]{2}{*}{$M$} & MEDIA & 30.10 & 39.85 & 24.13 & 36.85 & 25.97 \\
\hline & & $\begin{array}{l}\text { MEDIA- } \\
\text { NA }\end{array}$ & 30.10 & 39.85 & 24.13 & 36.85 & 25.97 \\
\hline \multirow[t]{4}{*}{19} & \multirow[t]{2}{*}{$\mathrm{F}$} & MEDIA & 19.40 & 22.45 & 16.50 & 31.56 & 30.10 \\
\hline & & $\begin{array}{l}\text { MEDIA- } \\
\text { NA }\end{array}$ & 19.15 & 30.35 & 18.63 & 34.99 & 31.59 \\
\hline & \multirow[t]{2}{*}{$M$} & MEDIA & 15.82 & 37.56 & 21.60 & 30.04 & 31.36 \\
\hline & & $\begin{array}{l}\text { MEDIA- } \\
\text { NA }\end{array}$ & 20.78 & 39.61 & 20.86 & 37.34 & 34.50 \\
\hline
\end{tabular}


Rangel Navia H , Pabón Villamizar J, Martinez Siza D, Contreras Suarez K.

\begin{tabular}{|c|c|c|c|c|c|c|c|}
\hline \multirow[t]{4}{*}{20} & \multirow[t]{2}{*}{$F$} & MEDIA & 24.07 & 23.98 & 23.33 & 30.43 & 31.04 \\
\hline & & $\begin{array}{l}\text { MEDIA- } \\
\text { NA }\end{array}$ & 22.97 & 25.53 & 21.18 & 30.52 & 28.65 \\
\hline & \multirow[t]{2}{*}{$M$} & MEDIA & 26.17 & 30.93 & 24.46 & 30.18 & 32.37 \\
\hline & & MEDINA & 27.83 & 25.40 & 24.06 & 28.46 & 31.36 \\
\hline \multirow[t]{4}{*}{21} & \multirow[t]{2}{*}{$\mathrm{F}$} & MEDIA & 24.26 & 26.80 & 22.25 & 32.69 & 32.37 \\
\hline & & $\begin{array}{l}\text { MEDIA- } \\
\text { NA }\end{array}$ & 23.20 & 29.78 & 23.09 & 33.30 & 33.28 \\
\hline & \multirow[t]{2}{*}{$M$} & MEDIA & 23.90 & 33.67 & 22.96 & 36.87 & 35.54 \\
\hline & & $\begin{array}{l}\text { MEDIA- } \\
\text { NA }\end{array}$ & 22.89 & 38.01 & 23.37 & 40.12 & 34.00 \\
\hline \multirow[t]{4}{*}{22} & \multirow[t]{2}{*}{$\mathrm{F}$} & MEDIA & No data & No data & No data & No data & No data \\
\hline & & $\begin{array}{l}\text { MEDIA- } \\
\text { NA }\end{array}$ & No data & No data & No data & No data & No data \\
\hline & \multirow[t]{2}{*}{$M$} & MEDIA & No data & No data & No data & No data & No data \\
\hline & & $\begin{array}{l}\text { MEDIA- } \\
\text { NA }\end{array}$ & No data & No data & No data & No data & No data \\
\hline \multirow[t]{4}{*}{23} & \multirow[t]{2}{*}{$F$} & MEDIA & 16.40 & 33.48 & 17.72 & 30.21 & 22.84 \\
\hline & & $\begin{array}{l}\text { MEDIA- } \\
\text { NA }\end{array}$ & 16.40 & 33.48 & 17.72 & 30.21 & 22.84 \\
\hline & \multirow[t]{2}{*}{$M$} & MEDIA & 28.36 & 27.13 & 28.525 & 29.438 & 41.695 \\
\hline & & $\begin{array}{l}\text { MEDIA- } \\
\text { NA }\end{array}$ & 28.36 & 27.13 & 28.525 & 29.438 & 41.695 \\
\hline \multirow[t]{4}{*}{24} & \multirow[t]{2}{*}{$F$} & MEDIA & 26.93 & 31.39 & 18.11 & 35.49 & 34.99 \\
\hline & & $\begin{array}{l}\text { MEDIA- } \\
\text { NA }\end{array}$ & 26.93 & 31.39 & 18.11 & 35.49 & 34.99 \\
\hline & \multirow[t]{2}{*}{$M$} & MEDIA & 17.937 & 71.409 & 34.138 & 26.037 & 29.408 \\
\hline & & $\begin{array}{l}\text { MEDIA- } \\
\text { NA }\end{array}$ & 17.937 & 71.409 & 34.138 & 26.037 & 29.408 \\
\hline \multirow[t]{4}{*}{25} & \multirow[t]{2}{*}{$\mathrm{F}$} & MEDIA & No data & No data & No data & No data & No data \\
\hline & & $\begin{array}{l}\text { MEDIA- } \\
\text { NA }\end{array}$ & No data & No data & No data & No data & no data \\
\hline & \multirow[t]{2}{*}{$M$} & MEDIA & 20.103 & 27.369 & 22.571 & 25.916 & 29.733 \\
\hline & & $\begin{array}{l}\text { MEDIA- } \\
\text { NA }\end{array}$ & 20.103 & 27.369 & 22.571 & 25.916 & 29.733 \\
\hline \multirow[t]{4}{*}{26} & \multirow[t]{2}{*}{$\mathrm{F}$} & MEDIA & 30.62 & 26.02 & 16.14 & 32.54 & 36.41 \\
\hline & & $\begin{array}{l}\text { MEDIA- } \\
\text { NA }\end{array}$ & 30.62 & 26.02 & 16.14 & 32.54 & 36.41 \\
\hline & \multirow[t]{2}{*}{$M$} & MEDIA & No data & No data & No data & No data & No data \\
\hline & & $\begin{array}{l}\text { MEDIA- } \\
\text { NA }\end{array}$ & No data & No data & No data & No data & No data \\
\hline
\end{tabular}

Fuente: Los autores

TABLA 5: resultados de la comparativa V.O.T SORDAS media, mediana según género y edad, de la oclusiva /p/ 


\begin{tabular}{|c|c|c|c|c|c|c|}
\hline \multicolumn{7}{|c|}{ V.O.T SORDOS } \\
\hline \multirow[t]{2}{*}{ EDAD } & \multirow[t]{2}{*}{ GENERO } & & \multicolumn{4}{|l|}{$\mathrm{P}$} \\
\hline & & & LAPIZ & PEINE & PIEDRA & ESPADA \\
\hline \multirow[t]{4}{*}{17} & \multirow[t]{2}{*}{$F$} & MEDIA & 11.82 & 15.46 & 15.09 & 13.00 \\
\hline & & $\begin{array}{l}\text { MEDIA- } \\
\text { NA }\end{array}$ & 11.82 & 15.46 & 15.09 & 13.00 \\
\hline & \multirow[t]{2}{*}{ M } & MEDIA & No data & No data & No data & No data \\
\hline & & $\begin{array}{l}\text { MEDIA- } \\
\text { NA }\end{array}$ & No data & No data & No data & No data \\
\hline \multirow[t]{4}{*}{18} & \multirow[t]{2}{*}{$\mathrm{F}$} & MEDIA & 23.63 & 14.52 & 17.68 & 13.89 \\
\hline & & $\begin{array}{l}\text { MEDIA- } \\
\text { NA }\end{array}$ & 25.45 & 14.21 & 16.81 & 11.79 \\
\hline & \multirow[t]{2}{*}{ M } & MEDIA & 14.85 & 17.64 & 26.35 & 15.80 \\
\hline & & $\begin{array}{l}\text { MEDIA- } \\
\text { NA }\end{array}$ & 14.85 & 17.64 & 26.35 & 15.80 \\
\hline \multirow[t]{4}{*}{19} & \multirow[t]{2}{*}{$\mathrm{F}$} & MEDIA & 17.73 & 14.88 & 20.04 & 15.78 \\
\hline & & $\begin{array}{l}\text { MEDIA- } \\
\text { NA }\end{array}$ & 17.60 & 15.17 & 19.75 & 15.53 \\
\hline & \multirow[t]{2}{*}{ M } & MEDIA & 17.11 & 15.05 & 14.21 & 16.10 \\
\hline & & $\begin{array}{l}\text { MEDIA- } \\
\text { NA }\end{array}$ & 17.42 & 14.73 & 13.15 & 17.76 \\
\hline \multirow[t]{4}{*}{20} & \multirow[t]{2}{*}{$F$} & MEDIA & 15.48 & 14.78 & 18.89 & 16.07 \\
\hline & & $\begin{array}{l}\text { MEDIA- } \\
\text { NA }\end{array}$ & 16.80 & 14.55 & 18.89 & 16.63 \\
\hline & \multirow[t]{2}{*}{$M$} & MEDIA & 15.53 & 14.95 & 18.05 & 17.69 \\
\hline & & MEDINA & 14.98 & 14.73 & 19.38 & 17.97 \\
\hline \multirow[t]{4}{*}{21} & \multirow[t]{2}{*}{$\mathrm{F}$} & MEDIA & 17.03 & 14.28 & 19.01 & 17.15 \\
\hline & & $\begin{array}{l}\text { MEDIA- } \\
\text { NA }\end{array}$ & 16.97 & 14.07 & 17.15 & 15.98 \\
\hline & \multirow[t]{2}{*}{$M$} & MEDIA & 18.20 & 14.45 & 16.09 & 16.89 \\
\hline & & $\begin{array}{l}\text { MEDIA- } \\
\text { NA }\end{array}$ & 17.55 & 13.84 & 14.01 & 16.87 \\
\hline \multirow[t]{4}{*}{22} & \multirow[t]{2}{*}{$\mathrm{F}$} & MEDIA & No data & No data & No data & No data \\
\hline & & $\begin{array}{l}\text { MEDIA- } \\
\text { NA }\end{array}$ & No data & No data & No data & No data \\
\hline & \multirow[t]{2}{*}{$M$} & MEDIA & No data & No data & No data & No data \\
\hline & & $\begin{array}{l}\text { MEDIA- } \\
\text { NA }\end{array}$ & No data & No data & No data & No data \\
\hline
\end{tabular}


Rangel Navia H, Pabón Villamizar J, Martinez Siza D, Contreras Suarez K.

\begin{tabular}{|c|c|c|c|c|c|c|}
\hline \multirow[t]{4}{*}{23} & \multirow[t]{2}{*}{$F$} & MEDIA & 17.93 & 16.85 & 23.54 & 15.34 \\
\hline & & $\begin{array}{l}\text { MEDIA- } \\
\text { NA }\end{array}$ & 17.93 & 16.85 & 23.54 & 15.34 \\
\hline & \multirow[t]{2}{*}{$M$} & MEDIA & 10.565 & 12.678 & 25.452 & 14.791 \\
\hline & & $\begin{array}{l}\text { MEDIA- } \\
\text { NA }\end{array}$ & 10.565 & 12.678 & 25.452 & 14.791 \\
\hline \multirow[t]{4}{*}{24} & \multirow[t]{2}{*}{$F$} & MEDIA & 17.27 & 13.97 & 18.33 & 15.99 \\
\hline & & $\begin{array}{l}\text { MEDIA- } \\
\text { NA }\end{array}$ & 17.27 & 13.97 & 18.33 & 15.99 \\
\hline & \multirow[t]{2}{*}{$M$} & MEDIA & 14.465 & 16.415 & 19.094 & 18.679 \\
\hline & & $\begin{array}{l}\text { MEDIA- } \\
\text { NA }\end{array}$ & 14.465 & 16.415 & 19.094 & 18.679 \\
\hline \multirow[t]{4}{*}{25} & \multirow[t]{2}{*}{$F$} & MEDIA & No data & No data & No data & No data \\
\hline & & $\begin{array}{l}\text { MEDIA- } \\
\text { NA }\end{array}$ & No data & No data & No data & No data \\
\hline & \multirow[t]{2}{*}{$M$} & MEDIA & 20.103 & 12.251 & 13.915 & 24.397 \\
\hline & & $\begin{array}{l}\text { MEDIA- } \\
\text { NA }\end{array}$ & 20.103 & 12.251 & 13.915 & 24.397 \\
\hline \multirow[t]{4}{*}{26} & \multirow[t]{2}{*}{$\mathrm{F}$} & MEDIA & 16.60 & 19.51 & 19.04 & 18.51 \\
\hline & & $\begin{array}{l}\text { MEDIA- } \\
\text { NA }\end{array}$ & 16.60 & 19.51 & 19.04 & 18.51 \\
\hline & \multirow[t]{2}{*}{$M$} & MEDIA & No data & No data & No data & No data \\
\hline & & $\begin{array}{l}\text { MEDIA- } \\
\text { NA }\end{array}$ & No data & No data & No data & No data \\
\hline
\end{tabular}

Fuente: Los autores

TABLA 6: resultados de la comparativa V.O.T SORDAS media, mediana según género y edad, de la oclusiva /t/.

\begin{tabular}{|c|c|c|c|c|c|c|c|c|c|}
\hline \multicolumn{10}{|l|}{ V.O.T } \\
\hline \multirow[t]{2}{*}{ EDAD } & \multirow{2}{*}{\multicolumn{2}{|c|}{$\begin{array}{l}\text { GE- } \\
\text { NE- } \\
\text { RO }\end{array}$}} & \multicolumn{7}{|l|}{$\mathrm{T}$} \\
\hline & & & TAZA & $\begin{array}{l}\text { TAM- } \\
\text { BOR }\end{array}$ & FRUTA & DIENTE & $\begin{array}{l}\text { CHA- } \\
\text { QUETA }\end{array}$ & $\begin{array}{l}\text { AUTO- } \\
\text { BÚS }\end{array}$ & CRISTAL \\
\hline \multirow[t]{4}{*}{17} & $\mathrm{~F}$ & $\begin{array}{l}\text { ME- } \\
\text { DIA }\end{array}$ & 14.18 & 17.73 & 17.82 & 16.55 & 27.19 & 16.91 & 15.37 \\
\hline & & $\begin{array}{l}\text { ME- } \\
\text { DIA- } \\
\text { NA }\end{array}$ & 14.18 & 17.73 & 17.82 & 16.55 & 27.19 & 16.91 & 15.37 \\
\hline & $M$ & $\begin{array}{l}\text { ME- } \\
\text { DIA }\end{array}$ & No data & No data & $\begin{array}{l}\text { No } \\
\text { data }\end{array}$ & No data & No data & $\begin{array}{l}\text { No } \\
\text { data }\end{array}$ & No data \\
\hline & & $\begin{array}{l}\text { ME- } \\
\text { DIA- } \\
\text { NA }\end{array}$ & No data & No data & $\begin{array}{l}\text { No } \\
\text { data }\end{array}$ & No data & No data & $\begin{array}{l}\text { No } \\
\text { data }\end{array}$ & No data \\
\hline
\end{tabular}




\begin{tabular}{|c|c|c|c|c|c|c|c|c|c|}
\hline \multirow[t]{4}{*}{18} & $F$ & $\begin{array}{l}\text { ME- } \\
\text { DIA }\end{array}$ & 15.25 & 18.05 & 13.63 & 17.66 & 16.10 & 13.76 & 19.26 \\
\hline & & $\begin{array}{l}\text { ME- } \\
\text { DIA- } \\
\text { NA }\end{array}$ & 13.26 & 19.02 & 12.85 & 16.36 & 16.62 & 12.95 & 20.26 \\
\hline & $M$ & $\begin{array}{l}\text { ME- } \\
\text { DIA }\end{array}$ & 17.45 & 14.34 & 13.63 & 21.74 & 18.72 & 16.95 & 19.26 \\
\hline & & $\begin{array}{l}\text { ME- } \\
\text { DIA- } \\
\text { NA }\end{array}$ & 17.45 & 14.34 & 12.85 & 21.74 & 18.72 & 16.95 & 20.26 \\
\hline \multirow[t]{4}{*}{19} & $\mathrm{~F}$ & $\begin{array}{l}\text { ME- } \\
\text { DIA }\end{array}$ & 18.67 & 15.53 & 12.38 & 16.96 & 17.06 & 13.16 & 18.05 \\
\hline & & $\begin{array}{l}\text { ME- } \\
\text { DIA- } \\
\text { NA }\end{array}$ & 17.18 & 15.01 & 12.62 & 16.51 & 19.38 & 12.90 & 15.55 \\
\hline & $M$ & $\begin{array}{l}\text { ME- } \\
\text { DIA }\end{array}$ & 12.88 & 15.98 & 13.55 & 19.74 & 16.20 & 14.29 & 19.22 \\
\hline & & $\begin{array}{l}\text { ME- } \\
\text { DIA- } \\
\text { NA }\end{array}$ & 13.47 & 17.42 & 13.94 & 17.98 & 12.27 & 13.24 & 19.78 \\
\hline \multirow[t]{4}{*}{20} & $\mathrm{~F}$ & $\begin{array}{l}\text { ME- } \\
\text { DIA }\end{array}$ & 15.94 & 16.29 & 13.96 & 19.14 & 14.06 & 16.53 & 16.69 \\
\hline & & $\begin{array}{l}\text { ME- } \\
\text { DIA- } \\
\text { NA }\end{array}$ & 15.84 & 16.80 & 13.01 & 18.20 & 14.23 & 16.57 & 16.17 \\
\hline & $M$ & $\begin{array}{l}\text { ME- } \\
\text { DIA }\end{array}$ & 17.35 & 15.45 & 13.43 & 15.83 & 14.76 & 14.97 & 19.04 \\
\hline & & $\begin{array}{l}\text { ME- } \\
\text { DINA }\end{array}$ & 14.98 & 13.74 & 13.69 & 16.03 & 11.51 & 14.98 & 19.47 \\
\hline \multirow[t]{4}{*}{21} & $\mathrm{~F}$ & $\begin{array}{l}\text { ME- } \\
\text { DIA }\end{array}$ & 17.56 & 15.58 & 12.59 & 19.70 & 12.54 & 16.48 & 16.37 \\
\hline & & $\begin{array}{l}\text { ME- } \\
\text { DIA- } \\
\text { NA }\end{array}$ & 17.72 & 15.90 & 13.68 & 18.86 & 14.03 & 23.12 & 16.37 \\
\hline & $M$ & $\begin{array}{l}\text { ME- } \\
\text { DIA }\end{array}$ & 19.46 & 17.10 & 12.32 & 19.04 & 20.74 & 16.88 & 17.98 \\
\hline & & $\begin{array}{l}\text { ME- } \\
\text { DIA- } \\
\text { NA }\end{array}$ & 17.93 & 17.23 & 12.96 & 18.98 & 20.67 & 17.60 & 18.24 \\
\hline
\end{tabular}


Rangel Navia H, Pabón Villamizar J, Martinez Siza D, Contreras Suarez K.

\begin{tabular}{|c|c|c|c|c|c|c|c|c|c|}
\hline \multirow[t]{4}{*}{22} & $\mathrm{~F}$ & $\begin{array}{l}\text { ME- } \\
\text { DIA }\end{array}$ & No data & No data & $\begin{array}{l}\text { No } \\
\text { data }\end{array}$ & No data & No data & $\begin{array}{l}\text { No } \\
\text { data }\end{array}$ & No data \\
\hline & & $\begin{array}{l}\text { ME- } \\
\text { DIA- } \\
\text { NA }\end{array}$ & No data & No data & $\begin{array}{l}\text { No } \\
\text { data }\end{array}$ & No data & No data & $\begin{array}{l}\text { No } \\
\text { data }\end{array}$ & No data \\
\hline & $M$ & $\begin{array}{l}\text { ME- } \\
\text { DIA }\end{array}$ & No data & No data & $\begin{array}{l}\text { No } \\
\text { data }\end{array}$ & No data & No data & $\begin{array}{l}\text { No } \\
\text { data }\end{array}$ & No data \\
\hline & & $\begin{array}{l}\text { ME- } \\
\text { DIA- } \\
\text { NA }\end{array}$ & No data & No data & $\begin{array}{l}\text { No } \\
\text { data }\end{array}$ & No data & No data & $\begin{array}{l}\text { No } \\
\text { data }\end{array}$ & No data \\
\hline \multirow[t]{4}{*}{23} & $\mathrm{~F}$ & $\begin{array}{l}\text { ME- } \\
\text { DIA }\end{array}$ & 11.84 & 17.35 & 11.43 & 17.10 & 13.82 & 18.34 & 13.93 \\
\hline & & $\begin{array}{l}\text { ME- } \\
\text { DIA- } \\
\text { NA }\end{array}$ & 11.84 & 17.35 & 11.43 & 17.10 & 13.82 & 18.34 & 13.93 \\
\hline & $M$ & $\begin{array}{l}\text { ME- } \\
\text { DIA }\end{array}$ & 11.074 & 11.624 & 15.356 & 19.017 & 14.791 & 16.904 & 14.791 \\
\hline & & $\begin{array}{l}\text { ME- } \\
\text { DIA- } \\
\text { NA }\end{array}$ & 11.074 & 11.624 & 15.356 & 19.017 & 14.79 & 16.904 & 14.791 \\
\hline \multirow[t]{4}{*}{24} & $\mathrm{~F}$ & $\begin{array}{l}\text { ME- } \\
\text { DIA }\end{array}$ & 11.14 & 13.62 & 15.39 & 14.82 & 17.31 & 12.67 & 18.72 \\
\hline & & $\begin{array}{l}\text { ME- } \\
\text { DIA- } \\
\text { NA }\end{array}$ & 11.14 & 13.62 & 15.39 & 14.82 & 17.31 & 12.67 & 18.72 \\
\hline & $M$ & $\begin{array}{l}\text { ME- } \\
\text { DIA }\end{array}$ & 11.572 & 12.151 & 12.094 & 18.57 & 11.527 & 14.466 & 14.465 \\
\hline & & $\begin{array}{l}\text { ME- } \\
\text { DIA- } \\
\text { NA }\end{array}$ & 11.572 & 12.151 & 12.094 & 18.57 & 11.527 & 14.466 & 14.465 \\
\hline \multirow[t]{4}{*}{25} & $\mathrm{~F}$ & $\begin{array}{l}\text { ME- } \\
\text { DIA }\end{array}$ & No data & No data & $\begin{array}{l}\text { No } \\
\text { data }\end{array}$ & No data & No data & $\begin{array}{l}\text { No } \\
\text { data }\end{array}$ & No data \\
\hline & & $\begin{array}{l}\text { ME- } \\
\text { DIA- } \\
\text { NA }\end{array}$ & No data & No data & $\begin{array}{l}\text { No } \\
\text { data }\end{array}$ & No data & No data & $\begin{array}{l}\text { No } \\
\text { data }\end{array}$ & No data \\
\hline & M & $\begin{array}{l}\text { ME- } \\
\text { DIA }\end{array}$ & 10.384 & 14.01 & 15.57 & 19.838 & 29.481 & 13.077 & 16.769 \\
\hline & & $\begin{array}{l}\text { ME- } \\
\text { DIA- } \\
\text { NA }\end{array}$ & 10.384 & 14.01 & 15.57 & 19.838 & 29.481 & 13.077 & 16.769 \\
\hline
\end{tabular}




\begin{tabular}{|llllllllll|}
\hline 26 & F & ME- & 15.96 & 15.54 & 15.37 & 16.44 & 19.74 & 17.99 & 9.31 \\
& DIA & & & & & & & \\
\cline { 2 - 9 } & ME- & 15.96 & 15.54 & 15.37 & 16.44 & 19.74 & 17.99 & 9.31 \\
& $\begin{array}{l}\text { DIA- } \\
\text { NA }\end{array}$ & & & & & & & \\
\hline M & ME- & No data & No data & No & No data & No data & No & No data \\
& DIA & & & data & & & data & \\
\cline { 2 - 9 } & ME- & No data & No data & No & No data & No data & No & No data \\
& DIA- & & & data & & & data & \\
NA & & & & & & & \\
\hline
\end{tabular}

Fuente: Los autores

TABLA 7: resultados de la comparativa V.O.T media, mediana según género y edad, de la oclusiva $/ \mathrm{T} /$.

\begin{tabular}{lllll}
\hline \multicolumn{5}{c}{ DDK- REPETICIONES - GENERAL } \\
\hline & /PA/ & /TA/ & /KA/ & /PATAKA/ \\
\hline MEDIA & 17.08 & 17.11 & 14.96 & 15.92 \\
\hline MEDIANA & 9 & 9 & 8 & 9 \\
\hline
\end{tabular}

Fuente: Los autores

TABLA 8: Resultados de media y mediana de DDK, repeticiones.

\begin{tabular}{lllll}
\hline \multicolumn{5}{c}{ DDK- TIEMPO - GENERAL } \\
\hline & /PA/ & /TA/ & /KA/ & /PATAKA/ \\
\hline MEDIA & 2.66 & 2.60 & 2.45 & 2.67 \\
\hline MEDIANA & 1.38 & 1.30 & 1.35 & 1.48 \\
\hline
\end{tabular}

Fuente: Los autores

TABLA 9: Resultados de media y mediana de DDK, tiempo.

\begin{tabular}{|c|c|c|c|c|c|c|}
\hline \multicolumn{7}{|c|}{ DDK - REPETICIONES } \\
\hline EDAD & GENERO & & /PA/ & /TA/ & /KA/ & /PATAKA/ \\
\hline \multirow[t]{4}{*}{17} & $\mathrm{~F}$ & MEDIA & 10 & 10 & 10 & 9 \\
\hline & & MEDIANA & 10 & 10 & 10 & 10 \\
\hline & M & MEDIA & No data & No data & No data & No data \\
\hline & & MEDIANA & No data & No data & No data & No data \\
\hline \multirow[t]{4}{*}{18} & $\mathrm{~F}$ & MEDIA & 9.42 & 8.21 & 9.00 & 8.40 \\
\hline & & MEDIANA & 7 & 7 & 7 & 9 \\
\hline & $M$ & MEDIA & 13.00 & 13.00 & 12.00 & 5.00 \\
\hline & & MEDIANA & 13 & 13 & 12 & 5 \\
\hline \multirow[t]{4}{*}{19} & $\mathrm{~F}$ & MEDIA & 8.00 & 8.00 & 8.00 & 7.50 \\
\hline & & MEDIANA & 8 & 8 & 8 & 9 \\
\hline & M & MEDIA & 16.68 & 13.95 & 11.97 & 6.23 \\
\hline & & MEDIANA & 7 & 7 & 7 & 3 \\
\hline
\end{tabular}




\begin{tabular}{|c|c|c|c|c|c|c|}
\hline \multirow[t]{4}{*}{20} & \multirow[t]{2}{*}{$\mathrm{F}$} & MEDIA & 8.50 & 7.50 & 7.00 & 6.00 \\
\hline & & MEDIANA & 8.5 & 7.5 & 7 & 6 \\
\hline & \multirow[t]{2}{*}{$M$} & MEDIA & 7.25 & 7.01 & 6.75 & 3.25 \\
\hline & & MEDIANA & 7 & 7 & 7 & 3 \\
\hline \multirow[t]{4}{*}{21} & \multirow[t]{2}{*}{$\mathrm{F}$} & MEDIA & 23.12 & 23.57 & 20.23 & 24.53 \\
\hline & & MEDIANA & 10 & 9 & 9 & 9 \\
\hline & \multirow[t]{2}{*}{$M$} & MEDIA & 18.31 & 19.02 & 17.16 & 17.25 \\
\hline & & MEDIANA & 13 & 9 & 9 & 9 \\
\hline \multirow[t]{4}{*}{22} & \multirow[t]{2}{*}{$\mathrm{F}$} & MEDIA & No data & No data & No data & No data \\
\hline & & MEDIANA & No data & No data & No data & No data \\
\hline & \multirow[t]{2}{*}{$M$} & MEDIA & No data & No data & No data & No data \\
\hline & & MEDIANA & No data & No data & No data & No data \\
\hline \multirow[t]{4}{*}{23} & \multirow[t]{2}{*}{$\mathrm{F}$} & MEDIA & No data & No data & No data & No data \\
\hline & & MEDIANA & No data & No data & No data & No data \\
\hline & \multirow[t]{2}{*}{$M$} & MEDIA & 21.00 & 17.00 & 17.00 & 9.00 \\
\hline & & MEDIANA & 21 & 17 & 17 & 9 \\
\hline \multirow[t]{4}{*}{25} & \multirow[t]{2}{*}{$\mathrm{F}$} & MEDIA & No data & No data & No data & No data \\
\hline & & MEDIANA & No data & No data & No data & No data \\
\hline & \multirow[t]{2}{*}{$M$} & MEDIA & 11.00 & 10.00 & 10.00 & 5.00 \\
\hline & & MEDIANA & 11 & 10 & 10 & 5 \\
\hline
\end{tabular}

Fuente: Los autores

TABLA 10: Resultados de la comparativa de DDK media y mediana según el género y la edad.

\begin{tabular}{|c|c|c|c|c|c|c|}
\hline \multicolumn{7}{|c|}{ DDK TIEMPO } \\
\hline EDAD & GENERO & & /PA/ & /TA/ & $/ \mathrm{KA} /$ & /PATAKA/ \\
\hline \multirow[t]{4}{*}{17} & $\mathrm{~F}$ & MEDIA & 1.67 & 1.58 & 1.53 & 1.33 \\
\hline & & MEDIANA & 1.67 & 1.58 & 1.53 & 1.33 \\
\hline & $M$ & MEDIA & No data & No data & No data & No data \\
\hline & & MEDIANA & No data & No data & No data & No data \\
\hline \multirow[t]{4}{*}{18} & $F$ & MEDIA & 1.55 & 1.39 & 1.27 & 1.87 \\
\hline & & MEDIANA & 1.70 & 1.31 & 1.00 & 1.77 \\
\hline & $M$ & MEDIA & 1.65 & 1.59 & 1.53 & 1.43 \\
\hline & & MEDIANA & 1.65 & 1.59 & 1.53 & 1.43 \\
\hline \multirow[t]{4}{*}{19} & $F$ & MEDIA & 1.11 & 1.13 & 1.19 & 1.34 \\
\hline & & MEDIANA & 1.08 & 1.11 & 1.17 & 1.33 \\
\hline & $M$ & MEDIA & 2.82 & 2.37 & 2.05 & 2.70 \\
\hline & & MEDIANA & 1.20 & 1.24 & 1.22 & 1.45 \\
\hline \multirow[t]{4}{*}{20} & $\mathrm{~F}$ & MEDIA & 1.36 & 1.23 & 1.26 & 1.49 \\
\hline & & MEDIANA & 1.36 & 1.23 & 1.26 & 1.49 \\
\hline & $M$ & MEDIA & 1.08 & 1.14 & 1.08 & 1.48 \\
\hline & & MEDIANA & 1.00 & 1.02 & 0.99 & 1.47 \\
\hline
\end{tabular}




\begin{tabular}{|c|c|c|c|c|c|c|}
\hline \multirow[t]{4}{*}{21} & \multirow[t]{2}{*}{$F$} & MEDIA & 3.70 & 3.54 & 3.40 & 3.68 \\
\hline & & MEDIANA & 1.43 & 1.33 & 1.41 & 1.59 \\
\hline & \multirow[t]{2}{*}{$M$} & MEDIA & 2.60 & 2.77 & 2.59 & 2.57 \\
\hline & & MEDIANA & 2.35 & 1.77 & 1.70 & 1.73 \\
\hline \multirow[t]{4}{*}{22} & \multirow[t]{2}{*}{$F$} & MEDIA & No data & No data & No data & No data \\
\hline & & MEDIANA & No data & No data & No data & No data \\
\hline & \multirow[t]{2}{*}{$M$} & MEDIA & No data & No data & No data & No data \\
\hline & & MEDIANA & No data & No data & No data & No data \\
\hline \multirow[t]{4}{*}{23} & \multirow[t]{2}{*}{$F$} & MEDIA & No data & No data & No data & No data \\
\hline & & MEDIANA & No data & No data & No data & No data \\
\hline & \multirow[t]{2}{*}{$M$} & MEDIA & 3.35 & 2.73 & 2.75 & 1.31 \\
\hline & & MEDIANA & 3.35 & 2.73 & 2.75 & 1.31 \\
\hline \multirow[t]{4}{*}{25} & \multirow[t]{2}{*}{$\mathrm{F}$} & MEDIA & No data & No data & No data & No data \\
\hline & & MEDIANA & No data & No data & No data & No data \\
\hline & \multirow[t]{2}{*}{$M$} & MEDIA & 1.40 & 1.30 & 1.28 & 2.02 \\
\hline & & MEDIANA & 1.40 & 1.30 & 1.28 & 2.02 \\
\hline
\end{tabular}

Fuente: Los autores

TABLA 11: Resultados de la comparativa de DDK media y mediana según el género y la edad.

(b) ACC VOT-DDK

La DDK no reporto resultados en el ACC, pues las correlaciones entre las variables; DDK-VOT; es alta y produce matrices singulares no positivas que no permite emplear la ACC. Sin embargo, se encontraron correlaciones canónicas entre VOT y las variables independientes (sexo, edad, nacimiento, procedencia), como se muestra en la Figura 01.
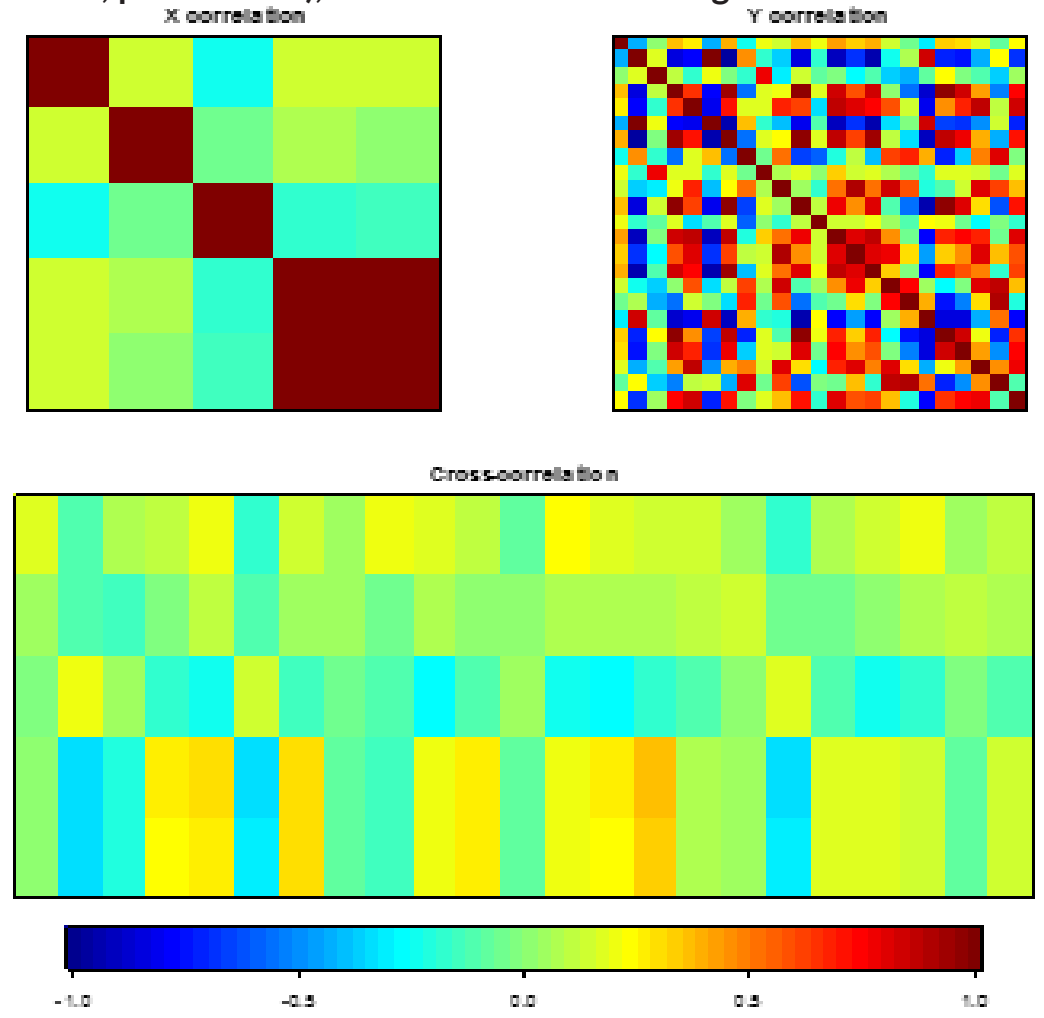

Figura 1. ANALISIS ESPECTRAL DE CORELACION CANONICA 
En la Figura 02, se observa que las dimensiones 1 y 2 de las correlaciones canónicas son las dominantes. Estas correlaciones se pueden observar en la Figura 03, donde es posible observar como la edad está fuertemente relacionada con VOT pero no ejerce ningún tipo de influencia sobre ella, a diferencia del sexo y los lugares de nacimiento y procedencia, que para la población de estudio fueron prácticamente los mismos.

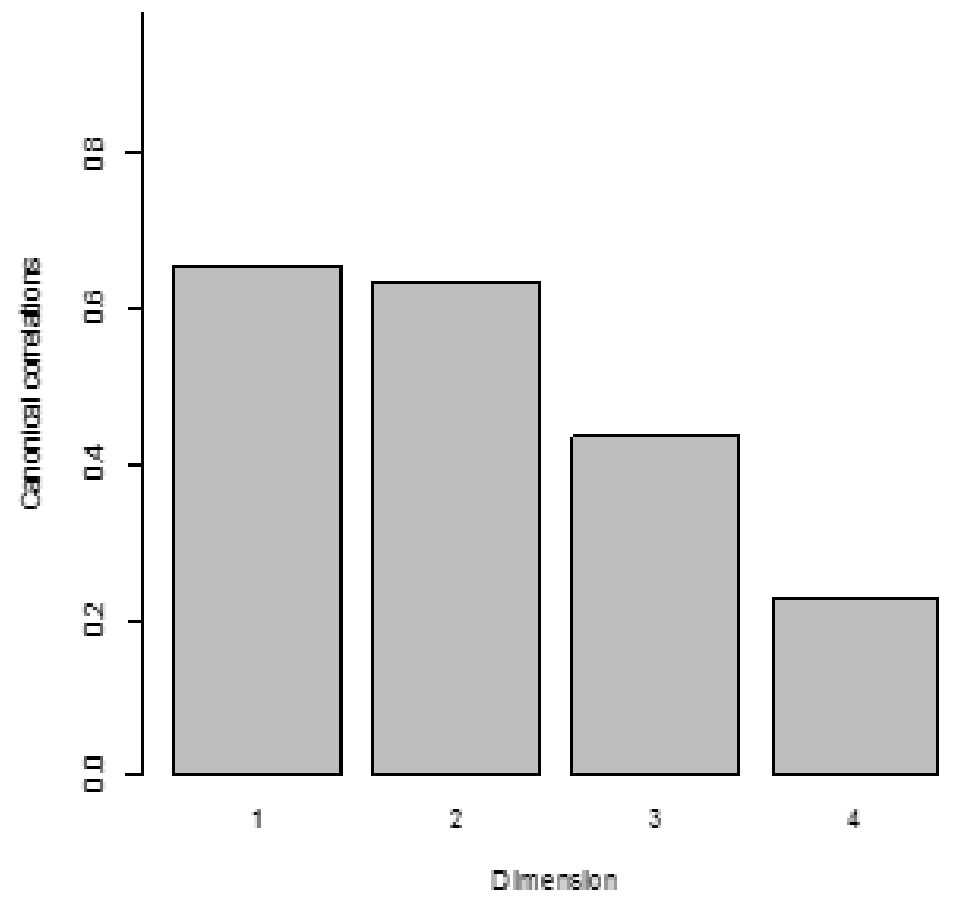

Figura 2.Dimensiones de correlación canónica

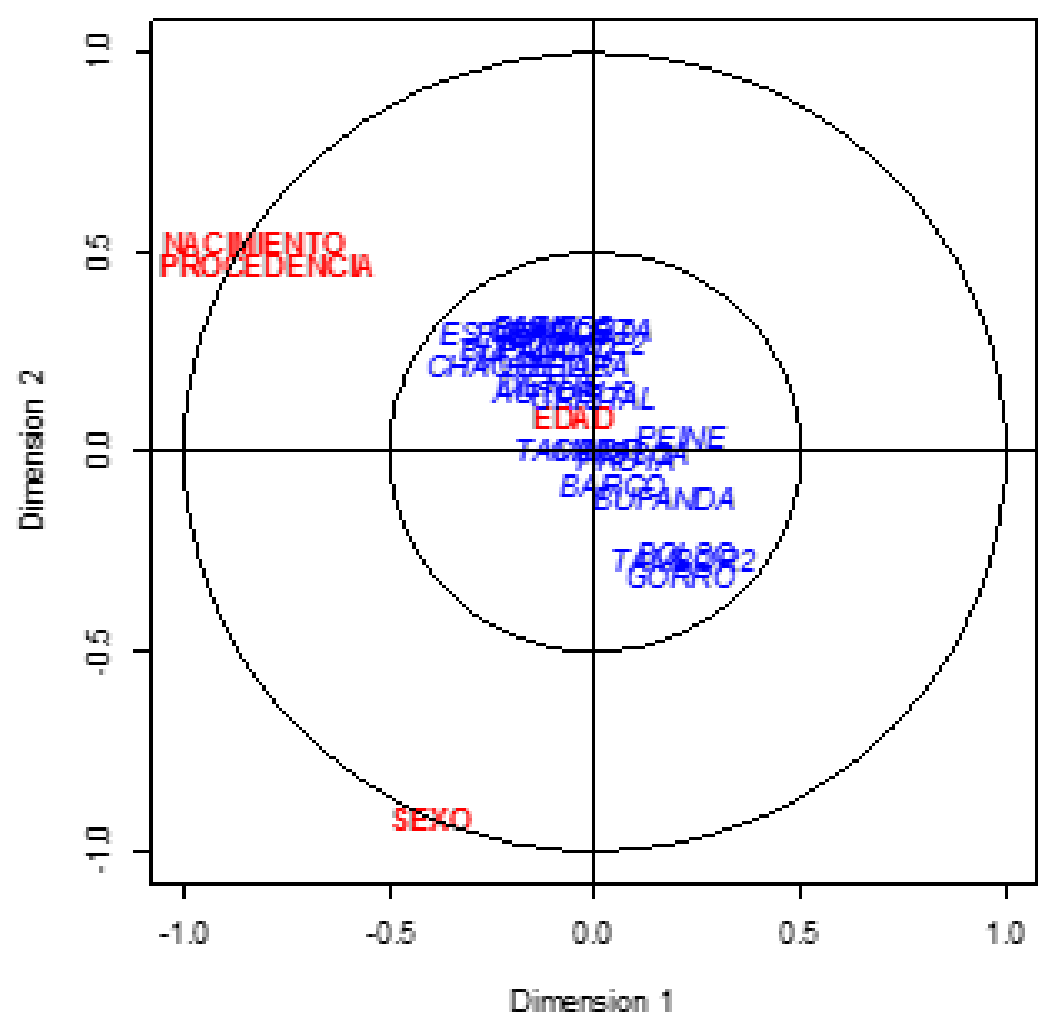

18 Figura 3. Plano cartesiano de correlación canónica 


\section{ANÁLISIS Y DISCUSIÓN}

Las líneas de análisis derivadas de los resultados se decantan en tres direcciones; (a) VOT; (b) DDK; (c) Relaciones estadísticas entre VOT y DDK; no obstante, todas ellas convergen en una línea de construcción de la evidencia; a saber; Procesos Diagnósticos en Desordenes Motores del Habla, referidos a los valores de referencia usados para determinar la presencia de alteraciones motrices sobre esta función; en este sentido las líneas argumentales del análisis son:

EI V.O.T como índice de diagnóstico para la disartria

EI V.O.T demuestra que no hay una relación constante entre los valores de cada par de fonemas de una misma zona de articulación, como los son /p-b-/, /d-t/, /k-g/ (1) siendo /p-t-k/ oclusivas sordas y /b-d-g/ oclusivas sonoras, (2) con estos fonemas se puede diagnosticar la disartria.

Se compararon los resultados de la investigación "medidas del vot para oclusivas españolas en estudiantes universitarios", para saber si se había alterado las referencias del diagnóstico de la disartria. (3)

\begin{tabular}{lll}
\hline & \multicolumn{3}{c}{ VOICE ONSET TIME } \\
\hline & REDIA RESPORTADA POR & MEDIA REPORTADA EN EL \\
& 15 & 16.5 \\
\hline TAZA & 18 & 22.2 \\
\hline MOSCA & 18 & 14.6 \\
\hline PEINE & 39 & 32.8 \\
\hline CHAQUETA 1 & 19 & 16.1 \\
\hline CHAQUETA 2 & 26 & 32.2 \\
\hline BARCO & 31 & 33.9 \\
\hline BLANCO & 15.6 & 16.4 \\
\hline ESPADA & 17.9 & 17.1 \\
\hline CRISTAL & 12 & 16.0 \\
\hline TAMBOR 1 & -18 & -47.1 \\
\hline TAMBOR 2 & 15.5 & 18.3 \\
\hline DIENTE & 18.5 & 18.4 \\
\hline PIEDRA & 17.8 & 17.7 \\
\hline FRUTA & 12.7 & 12.6 \\
\hline AUTOBUS & &
\end{tabular}

Fuente: Los autores

TABLA 12: Media reportada por Rangel; Torres y Mojica comparada con la reportada en el estudio.

En las tablas de comparación se ve un aumento en el valor de barco en la oclusiva sorda /k/ del estudio actual, con un aumento de $6.6 \mathrm{mls}$, , este evento podría explicarse como resultado de la relación canónica encontrada entre VOT y género, toda vez que el estudio reporte una relación moderada entre estas variables. En este sentido es probable que los efectos co-articulatorios de los sonidos / $\mathrm{k} /$ en posición silábica final y en relación con la vocal /o/ podrían explicar este fenómeno, En /p-t-k/ los valores aumentan cuanto más posterior sea la consonante; por el contrario, en /b-d-g/ los valores disminuyen cuanto más posterior sea la consonante. (1) Quiere decir que según sea la vocal acompañante puede aumentar el tiempo

En esta tabla se demuestra que el V.O.T de la vocal /o/ con la consonante /k/, coinciden con los 
reportes sobre el efecto co- articulatorio de las oclusivas (4) la cual arroja que los tiempos de barco se encuentra en un rango normal con una diferencia muy pequeña del dato de la tabla, Siendo así la coarticulación un determinante para los resultados del V.O.T.

Otro evento que podría explicar los cambios en el VOT es la composición sociofonética a partir de la cual es posible que nos permite diferenciar las voces de cada hablante y la lengua que es la que refleja las características del grupo social la cual pertenece los hablantes, tiene unos factores internos que motivan la variación fonológica como la edad, el sexo y el nivel socioeconómicos de los hablantes, evidenciando la relación de la sociofonética con el V.O.T. (5). Al comparar los resultados obtenidos en la investigación sigue mostrando unas referencias para Colombia.

\begin{tabular}{|c|c|c|c|}
\hline \multicolumn{4}{|c|}{ DIADOCOCINESIA } \\
\hline & & MEDIA REPORTADA & MEDIA REPORTADA \\
\hline & & $\begin{array}{l}\text { POR RANGEL, TO- } \\
\text { RRES, MOJICA }\end{array}$ & EN EL ESTUDIO \\
\hline \multirow[t]{3}{*}{ /PA/ } & REPETICIÓN & 58.70 & 17.08 \\
\hline & TIEMPO & 9.03 & 2.66 \\
\hline & $\mathrm{R} / \mathrm{T}$ & 6.50 & 6.41 \\
\hline \multirow[t]{3}{*}{ /TA/ } & REPETICIÓN & 61.05 & 17.11 \\
\hline & TIEMPO & 9.14 & 2.60 \\
\hline & $\mathrm{R} / \mathrm{T}$ & 6.68 & 6.58 \\
\hline \multirow[t]{3}{*}{ /KA/ } & REPETICIÓN & 53.24 & 14.94 \\
\hline & TIEMPO & 8.76 & 2.45 \\
\hline & $\mathrm{R} / \mathrm{T}$ & 6.09 & 6.11 \\
\hline \multirow[t]{3}{*}{ /PATAKA/ } & REPETICIÓN & 62.08 & 15.92 \\
\hline & TIEMPO & 8.54 & 2.67 \\
\hline & $\mathrm{R} / \mathrm{T}$ & 7.27 & 5.96 \\
\hline
\end{tabular}

Fuente: Los autores

Tabla 13. Media de DDK reportada por Rangel; Torres y Mojica comparada con la media de DDK reportada en el estudio.

En la investigación pasada se arrojaron valores de 5 a 7 repeticiones tomado como intervalo cada segundo. Comparado con los resultados producto de la investigación desarrollada actualmente, se obtuvo que la cantidad de repeticiones disminuyo considerablemente. (6)

Podríamos relacionar tal disminución con efectos asociados a factores como la articulación, ya que la integración de las estructuras involucradas en el habla como labios y lengua, definen el índice acústico de la velocidad de movimiento y la colocación articulatoria. El análisis acústico de la velocidad de estas transiciones es un procedimiento utilizado en la evaluación e investigación del habla disártrica, ya que aporta información sobre los movimientos de la lengua en la articulación. (7)

Así como la respiración, que en condiciones normales se realiza por vía nasal pero las dificultades presentadas en vías aéreas obligan al cuerpo a respirar por vía oral, estas condiciones pueden influir en la cantidad de repeticiones que puede hacer el paciente durante un intervalo de tiempo. (8)

Los resultados obtenidos señalan que estadísticamente no hay relación entre V.O.T y D.D.K; existen dos rutas posibles para explicar esta ausencia de relaciones entre voice onset time y diadococinecia como indices predictivos el uno del otro; a saber; por la diferencia en la tasa de 20 producción del habla que se relaciona estrictamente con los cambios encontrados en la compo- 
Análisis de correlación canónica entre voice onset time y diadococinesia del habla del español colombiano.

sición acústica de la articulación que genera el ritmo como indicador de tiempo.

Por efectos de la curva melódica que reflejan la composición temporal de la producción del habla

\section{CONCLUSIONES}

El aumento de V.O.T en la oclusiva /b/ y / k/ está relacionada con el contorno melódico como variable socio fonética. La caracterización de este efecto requiere mayores estudios.

Garantizar que el tiempo mínimo de la emisión de DDK sea igual o superior a 5 segundos comprende un requisito fundamental para la obtención de una buena muestra.

Gracias a los análisis encontrados en el estudio se demostró que no hay una relación predictiva entre vot y ddk; Sin embargo se encontró una relación entre el género y v.o.t como también una relación entre edad y v.o.t.

\section{TRABAJOS CITADOS}

1. Navia HJR, Barbosa ACT, Galeano BJM. MEDIDAS DE VOT PARA OCLUSIVAS ESPAÑOLAS EN ESTUDIANTES UNIVERSITARIOS: NUEVAS APROXIMACIONES DE ANALISIS. ReV CIENTÍFICA SIGNOS FÓNICOS [Internet]. 2016 Apr 1 [cited 2016 Nov 15];2(1). Available from: http://revistas.unipamplona.edu.co/ojs_viceinves/index.php/CDH/article/view/1843

2. J R, Cmejia R, Ruzickova H, Ruzicka E. Quantitative acoustic measurements for characterization of speech and voice disorders in early untreated Parkinson's disease. Journal of the Acoustical Society of America. 2011 Jan; 129(1): p. 350-367.

3. Kim Y, Kent R, Weismer G. An Acoustic Study of the Relationships Among Neurologic Disease, Dysarthria Type, and Severity of Dysarthria. Journal of Speech, Language, and Hearing Research. 2011; 54(2): p. 417-429.

4. Weismer G, Yunusova Y, Bunton K. Measures to Evaluate the Effects of DBS on Speech Production. Journal of neurolinguistics. 2012; 25(4): p. 74-94.

5. Ghioa A, Pouchoulina G, Testona B, Pintoa S, Fredouilleb C, De Loozea C, et al. How to manage sound, physiological and clinical data of 2500 dysphonic and dysarthric speakers? Speech Communication. 2012 Jun; 54(5): p. 664-679.

6. Navia HJR, Quintero NAG, Criollo PLN. DIADOCOCINESIA DEL HABLA EN ESTUDIANTES UNIVERSITARIOS. Rev CIENTÍFICA SIGNOS FÓNICOS [Internet]. 2016 Apr 1 [cited 2016 Nov 15];2(1). Available from: http://revistas.unipamplona.edu.co/ojs_viceinves/index.php/CDH/article/view/1786

7. Vardhan Sharma H, Hasegawa-Johnson M. Acoustic model adaptation using in-domain background models for dysarthric speech recognition. Computer Speech \& Language. 2013; 27(6): p. 1147-1162.

8. Yu V, Kadis D, Oh A, Goshulak D, Namasivayam A, Pukonem M, et al. Changes in voice onset time and motor speech skills in children following motor speech therapy: Evidence from/pa/productions. Clinical Linguistics \& Phonetics. 2014 Jan; 28(6). 
Rangel Navia H, Pabón Villamizar J, Martinez Siza D, Contreras Suarez K.

9. Orozco-Arroyave J, Arias-Londoño J, Vargas-Bonilla J, Gonzalez-Rátiva M, Nöth E. New Spanish speech corpus database for the analysis of people suffering from Parkinson's disease. In Proceedings of the Ninth International Conference on Language Resources and Evaluation; 2014: European Language Resources Association. p. 342-347.

10. Cmejla R, Rusz J, Bergl P, Vokral J. Bayesian changepoint detection for the automatic assessment of fluency and articulatory disorders. Speech Communication. 2013 Jan; 55(1): p. 178-189.

11. Lammert A, Goldstein L, Narayanan S, Iskarous K. Statistical methods for estimation of direct and differential kinematics of the vocal tract. Speech Communication. 2013 Jan; 55(1): p. 147161.

12. Okan Sakar O, Kursun OGF. Ensemble cannonical correlation analysis. Applied Intelligence. 2014 Feb; 40(2).

13. Yasna Roldán V., Jaime Soto-Barba. El V.O.T. de /p-t-k/ y /b-d-g/ en el español de Valdivia: un análisis acústico. Estudios filológicos. 1997.

14. Chela-Flores, Godsuno. Oclusivas, fricativas y aproximantes en el español: hacia una explicación de la marcadez y estado actual de las obstruyentes sonoras. 2009 agosto.

15. Cañas SJA, Redondo AL, Nieto ÁPA, Portilla EMP, Rangel RLE. METODOLOGIA: TAMIZ AUDITIVO NEONATAL. Rev CIENTÍFICA SIGNOS FÓNICOS [Internet]. 2016 Oct 25 [cited 2016 Nov 15];2(3). Available from: http://revistas.unipamplona.edu.co/ojs_viceinves/index.php/CDH/ article/view/2002

16. Nueva gramática de la lengua española. Fonética y fonología Barcelona: espasa libros 2009.

17. CARRASQUERO H., Viktor M. 2010 abril

18. Garcia MV, Niño APT, Castellanos MFI, Angulo LMA, Juliao ILL. DIGITALIZACIÓN DE PROTOCOLOS DE EVALUACIÓN EN EL ÁREA DE HABLA, MEDIANTE TECNOLOGÍAS DE DESARROLLO WEB Y APLICACIONES WINDOWS QUE CONTRIBUYAN A MEJORAR LA PRACTICIDAD DE LA APLICACIÓN. Rev CIENTÍFICA SIGNOS FÓNICOS [Internet]. 2016 Oct 25 [cited 2016 Nov 15];2(3). Available from: http://revistas.unipamplona.edu.co/ojs_viceinves/index.php/CDH/ article/view/2281

19. Relación entre las pendientes del segundo formante y las alteraciones motoras del habla en la disartria 2016 April volumen 36.

20. Alexander M. Gobermana, ?and Carl Coelhob. Acoustic analysis of Parkinsonian speech I:Speech characteristics and L-Dopa therapy 2002 January.

21. Redondo AL, Gallego CAM, Florez LLP, Machuca YGC, Mejia KLH, Jerez LVM, et al. RELACION ENTRE LA DISCAPACIDAD Y LA DESIGUALDAD DE LA CLASE SOCIAL. Rev CIENTÍFICA SIGNOS FÓNICOS [Internet]. 2016 Oct 25 [cited 2016 Nov 15];2(3). Available from:

http://revistas.unipamplona.edu.co/ojs_viceinves/index.php/CDH/article/view/2017 